\title{
Implementing a Standard DVB-T System using MATLAB Simulink
}

\author{
Mahmoud Elsharief \\ Electronic and communication, \\ Faculty of Engineering, Al-Azhar \\ University, Cairo, Egypt
}

\author{
AbdelHaliem Zekry \\ Electronic and communication, \\ Faculty of Engineering, Ain Shams \\ University, Cairo, Egypt
}

\author{
Mohamed Abouelatta \\ Electronic and communication, \\ Faculty of Engineering, Ain Shams \\ University, Cairo, Egypt
}

\begin{abstract}
This paper describes the Simulink implementation of all functional blocks of a standard Digital Video Broadcasting Terrestrial (DVB-T) transmitter and receiver and presents the simulation model for DVB-T system according to ETSI EN 300744 V1.6.1. The implemented model contains all channel coding and modulation building blocks with the parameters: $8 \mathrm{~K}$ OFDM-mode, code rate $(1 / 2,2 / 3$, and $3 / 4)$, and Modulation (64-QAM, 16-QAM). Realizing such system level implementation is required to study the effect of the channel on the transmission properties of the system and it helps getting the required quality of service by adjusting the system parameters. In addition, such model is the first step in software defined radio implementation. The bit error rate as a function of the signal to noise ratio $\mathrm{C} / \mathrm{N}$ is evaluated to be used in the link budget calculations.
\end{abstract}

\section{General Terms}

DSP System, DVB-T, Computer Applications

\section{Keywords}

Simulink implementation of DVB-T, COFDM, 64-QAM, 16QAM, Code rate, AWGN, MPEG-2

\section{INTRODUCTION}

DVB-T is a European-based consortium standard for the broadcast transmission of digital terrestrial television [1]. At present there is huge interest in DVB. This is because digital television can deliver vast amounts of information at very low cost to the maximum number of viewers. It can now be fully integrated into completely digital transmission networks [2]. This system transmits compressed digital video, audio and data, in a Moving Pictures Expert Group 2 (MPEG-2) transport stream as source coding, using Coded Orthogonal Frequency Division Multiplexing (COFDM) modulation [1].There are two mode OFDM frame structures to use for the $2 \mathrm{~K}$ and $8 \mathrm{~K}$ transmission modes [3]. There have been many publications in this area, covering different topics of the whole system as well as broadcasting network such as source coding for information compression and channel coding for error protection as in [1]. But system models do not include Energy dispersal and packetization building blocks. In this paper we construct a more comprehensive Simulink model. Such model is very required for software defined radio implementation of the system using either DSP or FPGA platforms; really this is our main intention. In addition, we can calculate the bit error rate as a function of the signal to noise ratio $\mathrm{C} / \mathrm{N}$ such curve is required for DTV radio design. The rest of the paper is organized as follows: section 2 describes the Simulink DVB-T transmitter model, section 3 presents the Simulink model of the DVB-T receiver, section 4 describes the simulation results and final section contains the conclusions.

\section{DESCRIPTION OF SIMULINK DVB-T TRANSMITTER}

Figure 1 shows the functional block diagram of the DVB-T transmitter. It consists of many signal processing blocks [3].

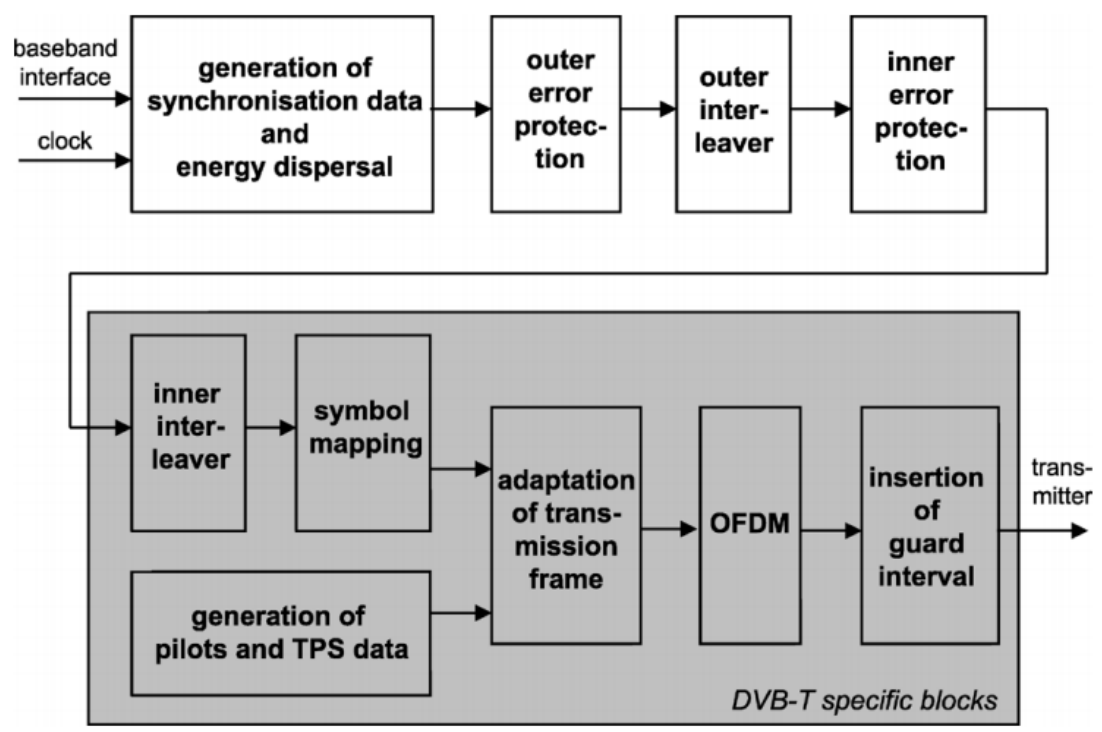

Fig 1: DVB-T transmitter signal processing blocks 


\subsection{Source coding and MPEG-2 multiplexing}

MPEG-2 is the standard developed for broadcasting applications and as a result it is used in all DVB transmissions. It is intended for application of high quality video images and audio signals encoding, with bit rates in the range of approximately $4-100$ Mbps. MPEG-2 transport streams (TS) includes many packetized MPEG-2 elementary streams (PES) of audio, video and private data [1]. Each TS Packet length is 188 bytes, 184 bytes are payload, and 4 bytes are headers. The first byte in header is SYNC word byte (i.e. 47HEX) [2, 3], Figure 2 shows the construction of the MPEG-2 packet. Source encoder compresses the original video into smaller bit rate. Video compression has two steps. The first is lossy using Discrete Cosine Transform (DCT) and the other is lossless compression based on variable length coding such as Huffman coding.

Fig 2: MPEG-2 transport streams (TS)

\begin{tabular}{|c|c|}
\hline SYNC & MPEG-2 Data \\
\hline & 187 Byte $\longrightarrow$ Byte $\longrightarrow$
\end{tabular}

\subsection{Multiplex adaptation and Randomization}

The aim of Randomization operation is to achieve a flat power-density spectrum and avoid occurrence of long strings 0 's and 1's. So the data must be randomized. MPEG-2 TS packets with length 187 data bytes plus one SYNC byte is XORed as shown in Figure 3 with the scrambler Pseudo-random binary sequence (PRBS) with generator polynomial $1+X^{14}+X^{15}$ and initial value 100101010000000 .

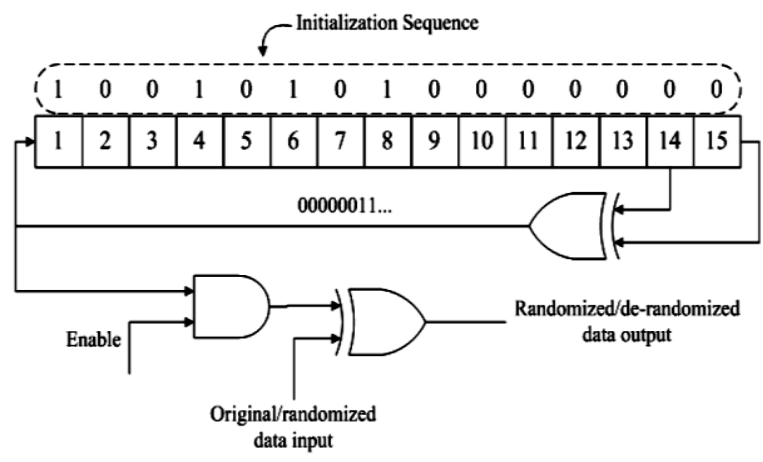

Fig 3: Scrambler/descrambler schematic diagram

The length of the PRBS is 1503 bytes. Each 8 MPEG-2 TS is combined to give a super frame of 1504 bytes according to Figure 4 . The first byte in a super frame $47_{\mathrm{HEX}}$ (SYNC) is inverted to became to $\mathrm{B} 8_{\mathrm{HEX}}(\mathrm{SYNC})$, while 1503 bytes is randomized.

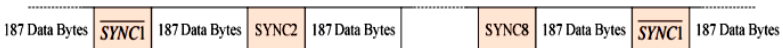

Fig 4: Randomized transport packets

\subsection{Outer Coder (Reed-Solomon Encoder)}

The output of the scrambler is delivered to a Reed-Solomon
The output of the scrambler is delivered to a Reed-Solomon outer channel encoder as shown in the block diagram of Figure 4. Blocks of data equal to 188 bytes' packets of MPEG-2 TS are applied to Reed-Solomon Encoder (RS) with the parameter:

The coded data length $=204$, the message length $\mathrm{k}=188$, and the number of correctable bytes $=8$ according to the standards depicted in Figure 5. Therefore, the output of RS encoder is 204 bytes [3]. RS adds 16 bytes which means that it can correct up to 8 errored bytes per MPEG-2 TS packet. Using RS, a bit error rate (BER) of $2 \times 10^{-4}$ can be achieved to obtain a quasi-errorfree $(\mathrm{QEF})$ data stream with residual BER of $10^{-11}$ [5]

\begin{tabular}{|c|c|c|}
\hline SYNC & MPEG-2 Data & Parity check \\
\hline
\end{tabular}

Fig 5: Reed-Solomon Encoder RS $(204,188, t=8)$

\subsection{Outer Interleaver (Convolutional Interleaver)}

The data obtained from the output of RS ( $N=204$ bytes) is applied to a convolutional Interleaver having the standard parameters: $(I=12)$ branches, and the depth $\mathrm{j} \times \mathrm{M}$ cells, Where $\mathrm{M}=\mathrm{N} / \mathrm{I}=17$, and $\mathrm{N}=204[3]$. A Convolutional interleaver rearranges the transport stream to increase the efficiency of the RS decoder [1]

\subsection{Inner Coder (Punctured Convolutional Encoder)}

The Punctured Convolutional Encoder is based on a mother Convolutional encoder of code rate $1 / 2$ and 64 states. The generator polynomials of the coder are $\mathrm{G} 1=171 \mathrm{OCT}$ for $\mathrm{X}$ output and G $2=1330 C T$ for $Y$ output. Puncturing technique is used to lower the redundancy of the mother code and obtain different code rates as $2 / 3,3 / 4,5 / 6$ and $7 / 8$ [3].

\subsection{Inner Interleaver}

The last process of these error protection techniques is the inner interleaver. Inner interleaver has two separate processes. Bit interleaver and symbol interleaver:

\subsubsection{Bit-wise interleaving}

Bit interleaving is performed only on the useful data. Data is demultiplexed into $\mathrm{v}$ sub-streams, where $\mathrm{v}=2,4$ and 6 for QPSK, 16-QAM, and 64-QAM respectively.

\subsubsection{Symbol Interleaver}

The aim of the symbol interleaver is to map v bit words onto the active carriers per OFDM symbol. 126 data from bit interleaver are grouped into 12 and 48 to give vector length 1512 and 6048 for $2 \mathrm{k}$ and $8 \mathrm{k}$ modes respectively.

\subsection{Signal Constellation Mapping} (Mapper)

The DVB-T systems use OFDM transmission. All data subcarriers in one OFDM symbol are modulated using QPSK, 16-QAM, 64-QAM uniform or non-uniform 16-QAM, and 64-QAM. In this paper, we implement as a model parameter uniform 64-QAM and uniform 16-QAM. The data stream output of symbol interleaver are mapped onto a complex number $z$, according to Figure 6. 


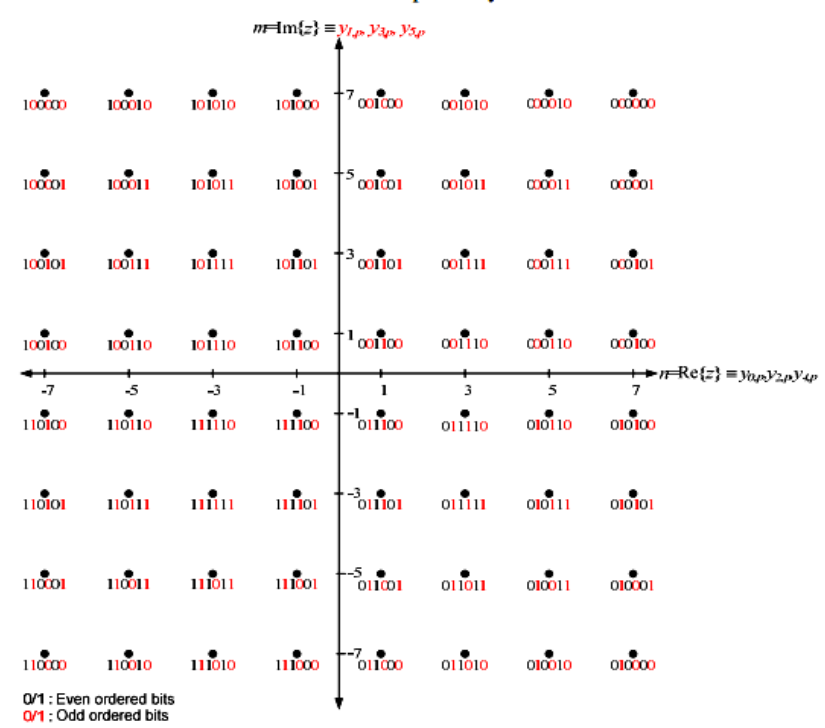

(a)

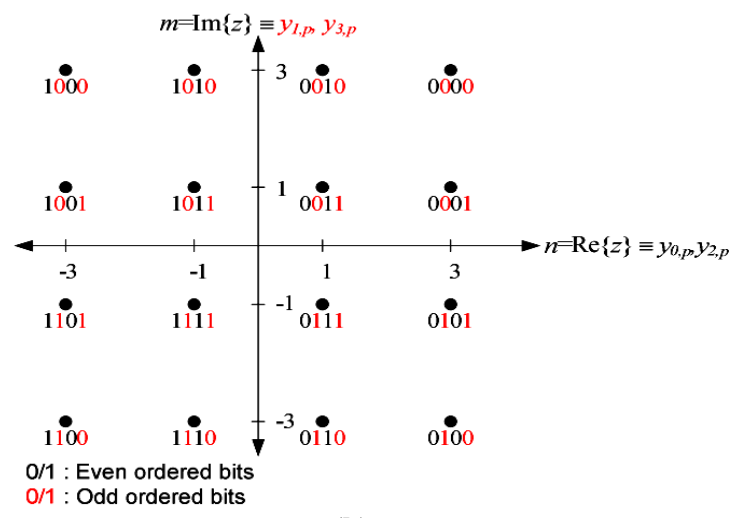

(b)

Fig 6: uniform mappings non-hierarchical, $\alpha=1$ (a) 64QAM (b) 16-QAM

\subsection{OFDM Transmitter and Guard}

\section{Interval Insertion}

The transmitted signal is organized in frames. Each frame consists of 68 symbols with duration TF. Each symbol is constituted by 6817 and 1705 carriers for $8 \mathrm{k}$ and $2 \mathrm{k}$ modes respectively. The duration of symbol Ts comprises two parts, a useful part TU, and a guard interval part $\Delta$. Guard interval takes one of four standard values: $1 / 4,1 / 8,1 / 16$, or $1 / 32$ from TU. Table 1 shows the different carriers assigned to different types of data in one OFDM symbol. After constructing the OFDM symbol in frequency domain, it is transformed into time domain using the Inverse fast Fourier transform (IFFT). As the length of IFFT sequence is $2 \mathrm{~K}$, which $\mathrm{k}$ is integer, we have to pad the sequences by zeros to complete the registry (2048 for $2 \mathrm{k}$ and 8192 for $8 \mathrm{k}$ ) [3, 4].

Table 1: Symbol transmitted of OFDM

\begin{tabular}{|c|c|c|}
\hline Number of & 2K & $\mathbf{8 K}$ \\
\hline Continual pilot & 45 & 177 \\
\hline Scattered pilot & 131 & 524 \\
\hline $\begin{array}{c}\text { Transmission parameter signaling (TPS) } \\
\text { carriers }\end{array}$ & 17 & 68 \\
\hline Data carriers & 1512 & 6048 \\
\hline
\end{tabular}

\section{DESCRIPTION OF SIMULINK DVB-T RECEIVER}

The receiving is not standardized like Transmission. However, the digital baseband DVB-T receiver strictly performs the inverse processes of that of the baseband transmitter. Hence, it consists of following signal processing blocks arranged from the input to the output:

- OFDM Receiver and Guard Interval removing

- Demapper

- Inner Deinterleaver

- Inner decoder (Viterbi Decoder)

- Outer Deinterleaver (Convolutional Deinterleaver $\mathrm{I}=12$ )

- Outer Decoder (Reed-Solomon Decoder)

- Demultiplex adaptation and Derandomization (Descrambler)

- MPEG-2 Demultiplexing and Source Decoding These processes are introduced in the next section.

\section{SIMULINK MODEL OF DVB-T SYSTEM AND SIMULATION RESULTS}

We built a Simulink model of the DVBT system based on the previous description of the system and the relevant standards. In this model we have chosen the following parameters of the different building blocks: $8 \mathrm{k}$ OFDM mode, code rate $=(1 / 2$, $2 / 3$ and 3/4), modulation type 64-QAM and 16-QAM, $\Delta=(1 / 4) T_{U}$, and Gaussian channel. System models with other parameters can be built in the same way. The implemented MATLAB SIMULINK model is shown in Figure 7. Simulation is based on an implemented simulation model in MATLAB m-file ((command "commdvbt"), 2k mode) [9]. Figure 8 shows the detailed building blocks representing the macroblock DVB-T $8 \mathrm{k}$ in Figure 8 . The model is validated by the loop back test where the output data from the receiver is found to be identical to the input data to the transmitter. Also Figure 9 shows the transmitted video and received video, both are similar.

Snap shots of the signal at the output of the different building blocks are shown in Figures 10 and 11. Figure 10 shows the constellations diagram of 64-QAM and 16-QAM signal after OFDM receiver demodulation. Also the effect of the white noise causes the scattering of the constellation points around their initial regular positions. However, there is no overlap of scatter plot such that one can correctly decide on the QAM symbols.

Simulation shows the spectrum of signal after transmissions in Figure 11. Figure 11 shows the output of the spectrum analyzer connected at the output of the transmission channel in Figure 8 to display the frequency spectrum of the received signal at the input of the receiver. The bandwidth of this signal amounts to $8 \mathrm{MHz}$ in agreement with the bandwidth of the baseband signal.

We studied the effect of the different channel coding on the error performance of the system. Therefore the BER is calculated as a function of the signal to noise ratio $\mathrm{C} / \mathrm{N}$ after inner Viterbi decoder, as well as outer RS decoder. 


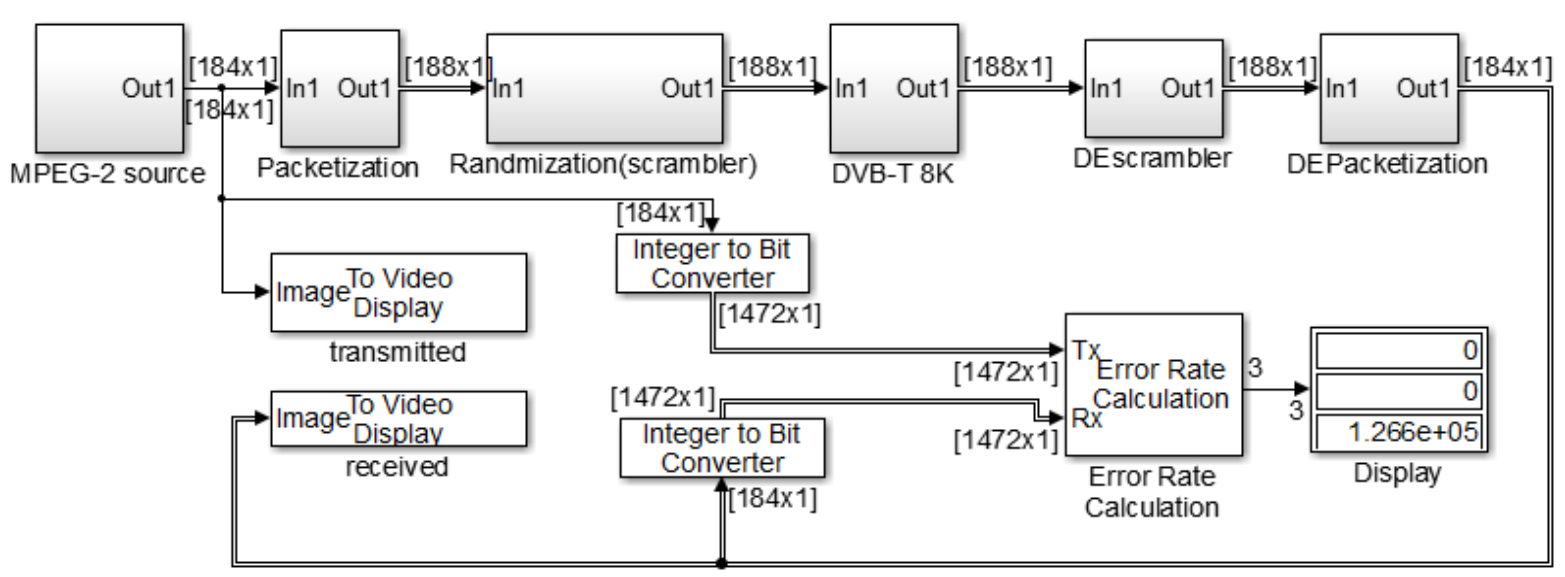

Fig 7: Digital Video Broadcasting Terrestrial 8K

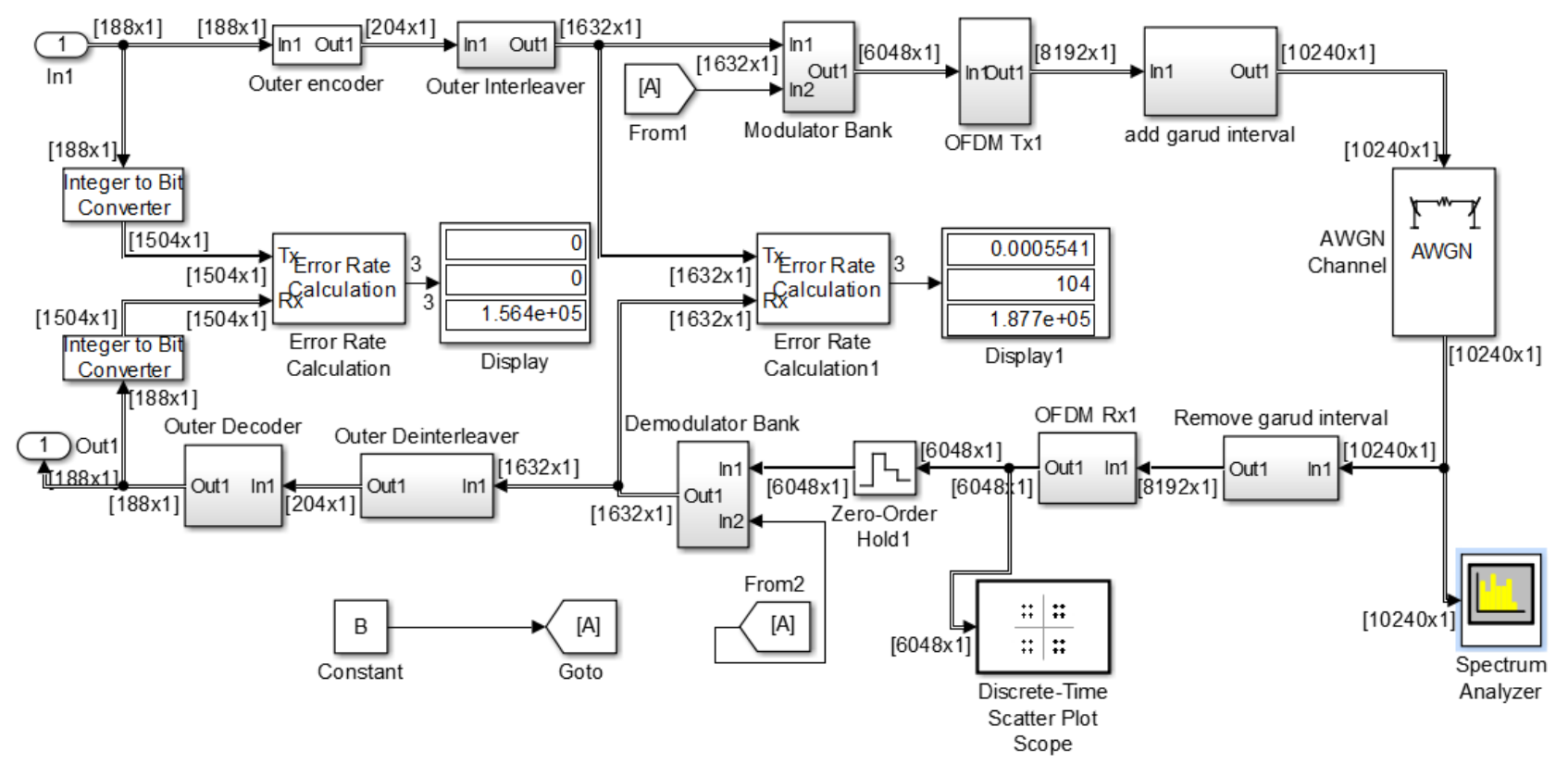

Fig 8: Detailed building blocks representing the macroblock DVB-T 8k

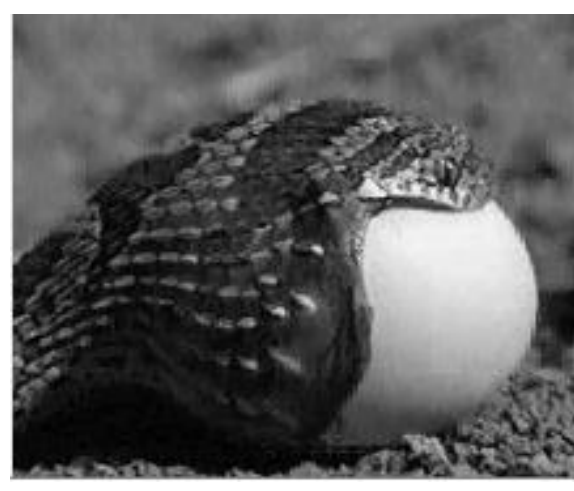

(a)

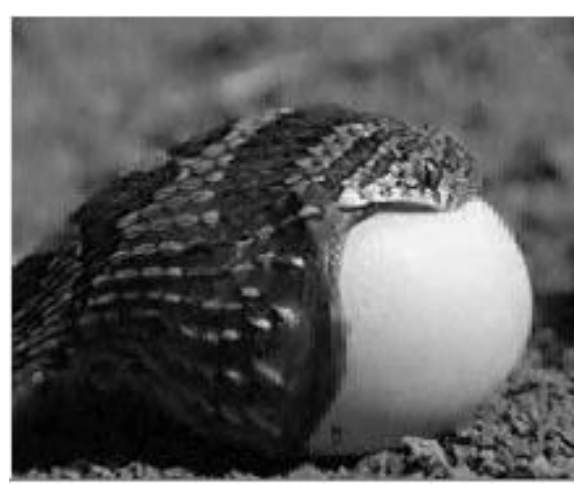

(b)

Fig 9: transmitted and received videos (a) original video (b) received video

The results are depicted in Figure 12 and Figure 13. We see from these figures that The BER decreases with the channel decoding for the same $\mathrm{C} / \mathrm{N}$ at the higher values of the signal to noise ratio. The BER after RS decoder should QEF (Quasi Error Free) when The BER after Viterbi decoder Reach to
$2 \times 10^{-4}$ according to standard [3]. Which is satisfied as is clear from Figures 12 and 13. Table 2 show final results compared to the results with ETSI standard. There an improving in SNR 


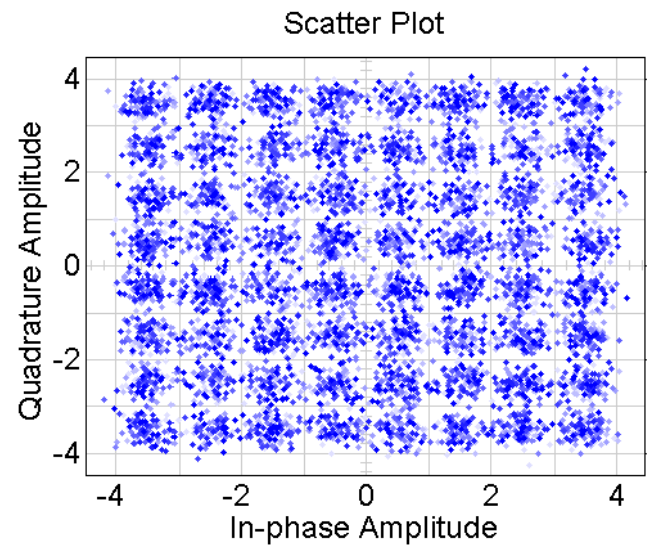

(a)

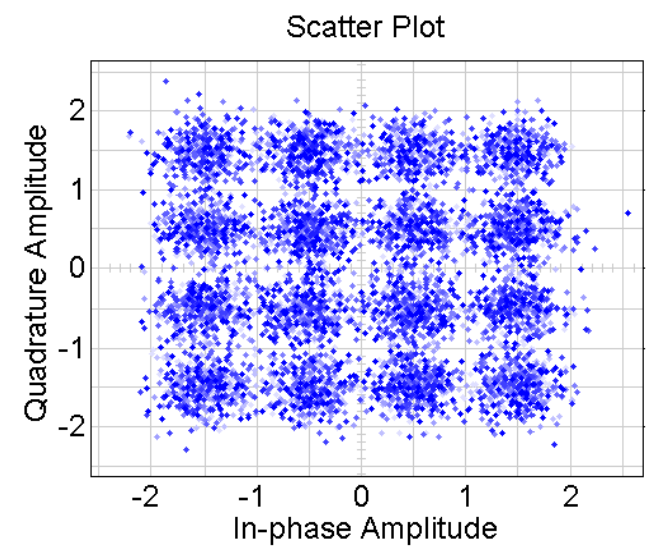

(b)

Fig 10: constellation diagram (a) 64-QAM (b) 16-QAM

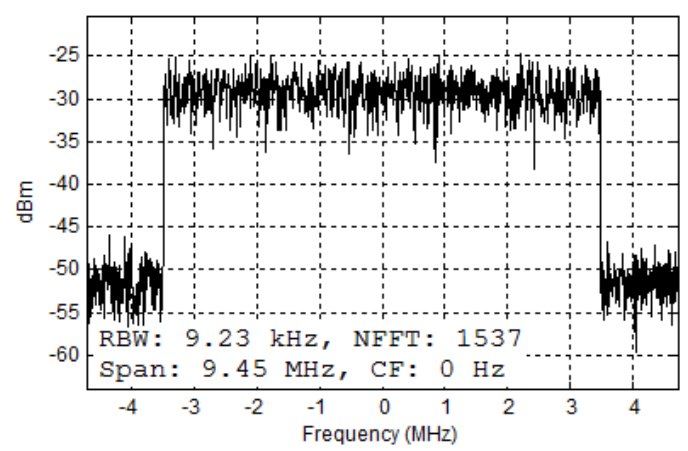

(a)

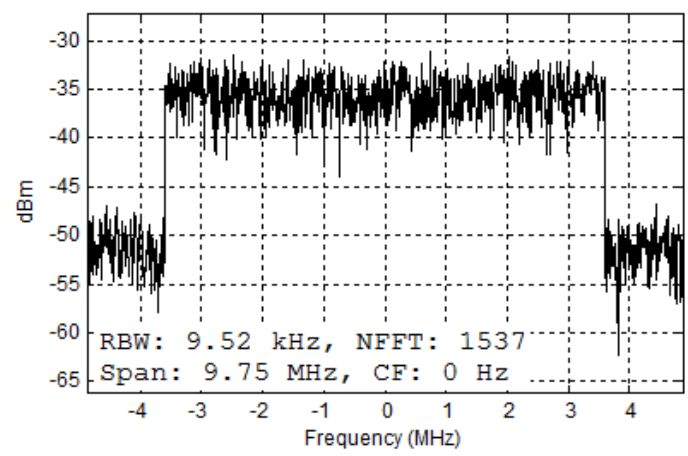

(b)

Fig 11: spectrum of signal after transmission (a) 64-QAM (b) 16-QAM

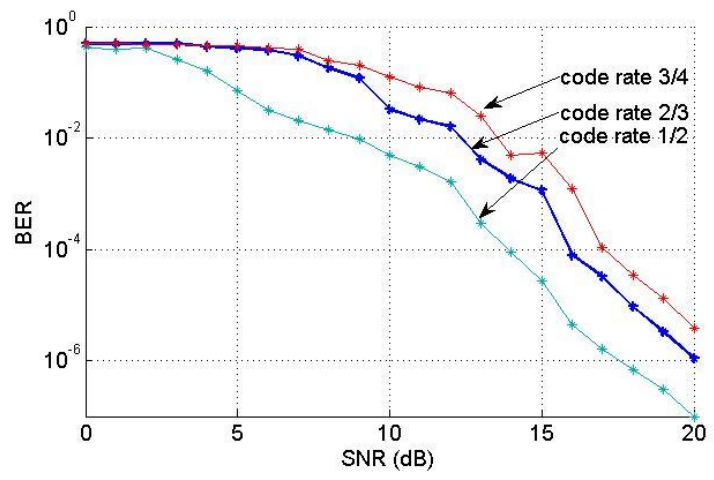

(a)

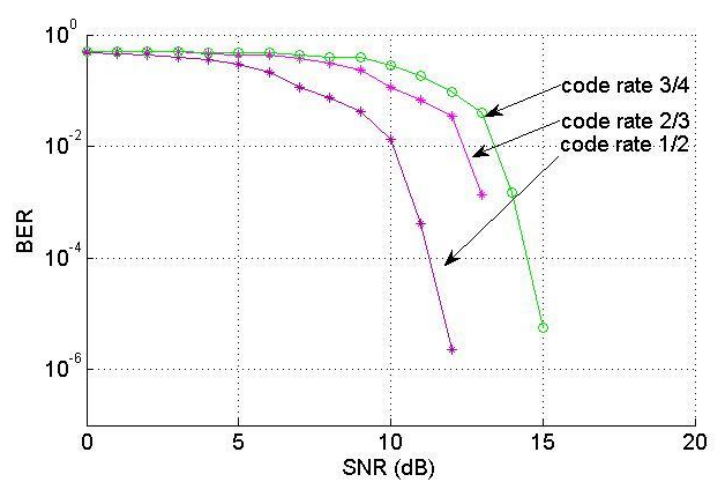

(b)

Fig 12: BER Verses SNR for 64-QAM (a) after viterbi decoder (b) after RS decoder

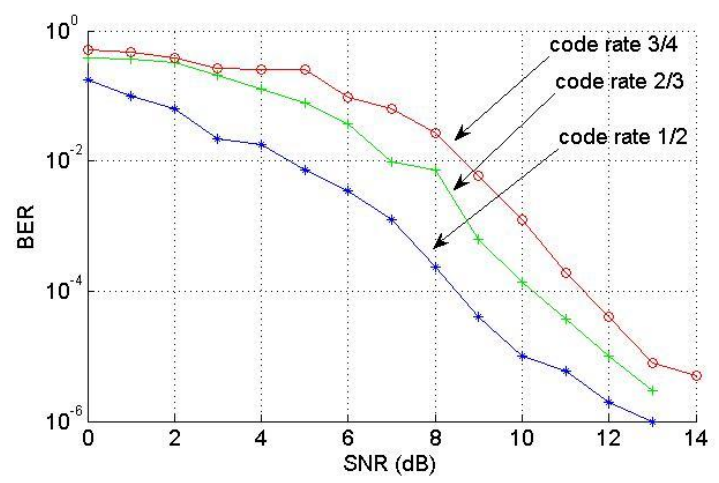

(a)

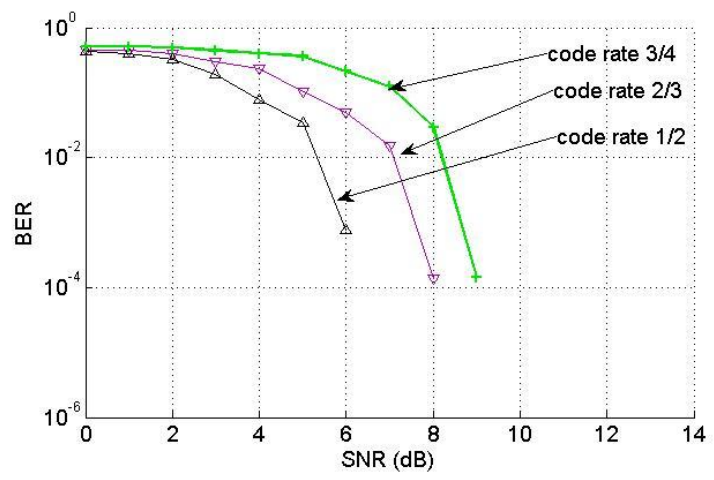

(b)

Fig 13: BER Verses SNR for 16-QAM (a) after viterbi decoder (b) after RS decoder 
Table 2: Required SNR (C/N) for Non-Hierarchical transmission to achieve a $B E R=2 \times 10-4$ after the Viterbi decoder using (AWGN and $\Delta=(1 / 4)$ TU)

\begin{tabular}{|c|c|c|c|c|c|}
\hline $\begin{array}{c}\text { Constell } \\
\text { ation }\end{array}$ & $\begin{array}{c}\text { Code } \\
\text { rate }\end{array}$ & $\begin{array}{c}\text { (SNR) } \\
\mathbf{d B}\end{array}$ & $\begin{array}{c}\text { ETSI } \\
\text { Stand } \\
\text { ard } \\
\text { (SNR) } \\
\mathbf{d B}\end{array}$ & $\begin{array}{c}\text { Enhanc } \\
\text { ement } \\
\mathbf{d B}\end{array}$ & $\begin{array}{c}\text { Percent } \\
\text { age } \\
\text { \% }\end{array}$ \\
\hline 16-QAM & $1 / 2$ & 8 & 9.3 & 1.3 & 13 \\
\hline 16-QAM & $2 / 3$ & 9.8 & 11.4 & 1.6 & 14 \\
\hline 16-QAM & $3 / 4$ & 11 & 12.6 & 1.6 & 12.5 \\
\hline 64-QAM & $1 / 2$ & 13 & 13.8 & .8 & 5.7 \\
\hline 64-QAM & $2 / 3$ & 15.5 & 16.7 & 1.2 & 7.1 \\
\hline 64-QAM & $3 / 4$ & 16.5 & 18.2 & 1.7 & 9.3 \\
\hline
\end{tabular}

\section{CONCLUSIONS}

In this paper we presented a matlab Simulink model for the most evolving digital video broadcasting system DVB-T including both the transmitter and the receiver. The developed model is verified by intensive testing of the building blocks and the integrated system. Simulink models are characterized by graphical user interface with easy understanding and altering the model to cope with the evolution of the standards. The built model can be considered as the first but the important step in software defined radio implementations because of the presence of powerful compilation tools from Simulink to either FPGA or DSPs or both. In addition the developed model can be used as an educational tool to explore the digital broadcast systems. The developed model is comprehensive except the source codec. The video codec will be developed and included to our model to get a complete model of DVB-T.

\section{REFERENCES}

[1] Dumic, E., Grgic, S., \&Sisul, G. 2010, September. Simulation model for the evaluation of DVB-T system parameters. In ELMAR, 2010 PROCEEDINGS (pp. 225230). IEEE

[2] O'Leary, S. 2000. Understanding digital terrestrial broadcasting. Artech House.

[3] ETSI, E. 300744 V1. 6.1 2008-09: European Standard (Telecommunications series) Digital Video Broadcasting (DVB). Framing structure, channel coding and modulation for digital terrestrial television.

[4] Zakaria, M. 2008. Performance Evaluation of DVB-H Systems, dissertation, ASU, Cairo, 2008

[5] Grunwald, S. 2001. Digital TV Rigs and Recipes Part 4 DVB-T. Rohde \& Schwarz Broadcasting Division, Publicación (febrero 2001).

[6] Brice, R. 2002. Newnes guide to digital TV. Newnes.

[7] Thyagarajan, K. S. 2011. Still Image and video compression with MATLAB. John Wiley \& Sons.

[8] Hanna, A. G. R., \&Zekry, A. H. (2012, February). Development of Wimax Physical Layer Building Blocks. In Intelligent Systems, Modelling and Simulation (ISMS), 2012 Third International Conference on (pp. 537-541). IEEE.

[9] Kotak, N. A., Sedani, B. S., \&Dwivedi, V. V. 2011, October. Simulation \& Performance Analysis of DVB-T System Using Efficient Wireless Channels. In Computational Intelligence and Communication Networks (CICN), 2011 International Conference on (pp. 677-681). IEEE. 